\title{
A Study on Computer-aided Psychological Measurement
}

\author{
Huajie Sui ${ }^{1}$, Xunbing Shen ${ }^{2 *}$ \\ ${ }^{1,2}$ School of Humanities, Jiangxi University of Traditional, Chinese Medicine,Nanchang,330001, \\ China \\ *Corresponding author
}

Key words: Psychology, computer, summary.

\begin{abstract}
The development of computer technology provides necessary technical support to the development and reform of psychological measurement. Analyzing the features and contents of computer-aided psychological measurement will push forward the research, development and innovation of computer-aided functions, and help perfect the existing technologies. The application of computer technology in psychological measurement pushes the research to enter a development field, greatly contributing to improving the quality and speed of psychological measurement.
\end{abstract}

\section{Introduction}

Under the current social context, scientific and technological development influences our life in every aspect. Especially since 1960, as computer and the Internet technology developed fast, social life has changed dramatically. In psychological study, computer information technology has been made full use of to substitute the pen and paper-based test method. Psychologists gradually turn to computer information technology for psychological measurement, from developing test questions to implementing psychological test, and to interpreting, grading and reporting after test. Through decades of development, computer technology has been widely used in many aspects of social life. The advantage of computer technology in computing is prominent, so that computer technology is used in the field of psychological measurement on a large scale, mainly covering test questions and questionnaires, etc. Thanks to the advantages of huge information, computational accuracy, and quick computation, computer technology plays a significant role in psychological counseling, personnel selection, mental ability exercise, and research education.

\section{Basic Researches on Computer-aided Psychological Measurement}

Computer was used in Strong Interest Test for scoring and computing by a famous American engineer Hanks in 1946 for the first time, which ushered in a new approach of psychological measurement. A computer-aided psychological measurement software with the function of interpreting results was used in the Mayo Psychological Measurement Studio in 1965 for the first time, and this software was mainly used in personality surveys and tests at the University of Minnesota. Since 1980, computer-aided psychological measurement software and the interpretation function had been developed rapidly in America and European countries. In 1988, there were 360 psychological measurement software registered with American Psychological Association, which just accounted for a small proportion at that time. According to related data statistics, the number of computer-aided measurement software used in children's personality test in America in 1990 reached 34. Since 1990, computer-aided psychological measurement had completely the pen and paper-based measurement method, and computer had gradually become a common tool in psychology, and expanded the measurement method in psychology, having a broad development prospect. After this, 
with the emergence and growth of the Internet, online hearing test was carried out by Welch depending on Internet connection in 1995 for the first time. With the rapid development and wide use of computer and Internet technology, psychological study develops rapidly, and psychological test enters people’s life.

\section{Application of Computer in Psychological Measurement}

In respect of the practicability and convenience, the application of computer in psychological measurement is mainly realized in two ways: compiling "computer-based test", in which computer is used as a media; compiling "self-adapting computer test", in which computer plays a decisive role. Besides, in psychological test researches, mathematical statistics, multi-media and other computer technologies are widely used.

\section{Computer-based Test}

In computer-based test, the main functions of computer are as follows. 1. Question bank: For a test, questions of different versions should be included in the test question bank. Test developer, when building a mega-question bank, must classify questions by the difficulty and content and store them in computer, and then different question banks will be produced based on the needs of those in need. 2. Computer test: Presently, computer test has completely substituted the pen and paper-based test. After the testee inputs his personal information, computer displays related test questions in order, and the testee answers the questions. 3. Automatic scoring: Computer will automatically score, then tabulate the result, and display the test results, which substitutes the compute mode with the aid of a scoreboard. 4. Analysis and result interpretation: According to the score of the testee, computer automatically inputs the score into the "expert system" to perform professional analysis on the result of the testee, and gives corresponding interpretation and training plan on this basis.

\section{Self-adapting Computer Test}

Self-adapting computer test is a new test method. Since 1950, the adaptive theory had been earnestly studied. In this method, computer is able to judge the actual ability of the testee, and search and designate test questions from the question bank suitable for the testee in respect of difficulty. After the testee finishes a question, computer will go on based on the reflection of the testee, and judge the information of the testee from time to time, so that an accurate test result will be achieved. Therefore, in test with such a method, the precision and accuracy can be strictly controlled. Self-adapting test is of high flexibility. Hence, it is widely used.

With the help of related software, researchers can call the test results at any time, modify, edit, add or delete test data, and combine the test data with that stored in other computers. In the processes of revising and compiling test, the norm data of test should be computed, verified and analyzed, to present complex statistical data via computer technology.

\section{Relationship with Traditional Test}

Computer greatly facilitates psychological test, but the scheme of pen and paper-based test differs from the method of computer-aided test, and the results obtained with the two methods are incomparable. Even in the event of the same questions, it cannot be judged that the reliability and difficulty of test questions displayed in computer remain unchanged. For example, Muddux believed that computer-aided test and pen and paper-based test were equal after making comparisons, but there were reports insisting that the two kinds of test were different. Snider found the reports insisting that computer-aided test and pen and paper-based test were equal to be unreasonable. Since personality assessment was not considered, this opinion of equal was ungrounded. In 1986, a policy specific to psychological research was formulated in America: Whether the computer-aided test conforms with the pen and paper-based test must be figured out for every case, the two methods should not be judged 
to be identical at will. Hence, as the test mode changes, researcher should change his research method. It's worth noting that the test tests must be comparable.

\section{Features of Computer-aided Psychological Measurement}

\section{Advantages of Computer-aided Psychological Measurement}

\section{Continuous Test Revision}

Continuous test revision mainly refers to: combining experimental test with actual test for continuous test as a system. The test process includes test revision. The revision of new test items must be based on the old items. As long as the specifications are met with, the new items can be included into the question bank. This revision method is highly demanding for system acquisition and data processing, so the data transmission and processing speed of computer is very critical. The United States Military has successfully developed a set of "Military Service Vocational Ability Test System", having realized continuous revision throughout test, which cannot be achieved in traditional pen and paper-based test.

\section{Advantages of Test Implementation}

A higher level of standardization can be realized in test; related test questions can be adjusted according to the characteristics of individuals from time to time; if the testee gives a wrong answer, a simpler question will be presented; otherwise, if the testee gives a right answer, a harder question will be presented; in this way, the testee can find test questions suitable for himself under the guidance of computer; computer-aided psychological measurement is not subject to human's mood, concentration level and memory ability; individual information excluded from the test also can be recorded, such as time consumption, which cannot be realized in pen and paper-based test, let alone in collective test; questions in the form of words, figure and even video can be displayed via computer, which facilitates and diversifies test. According to the research of Dignon, 85\% psychological testees tend to accept computer-aided psychological measurement, and reject psychologist-aided measurement. Besides, computer is unlimitedly tolerable, especially suitable for schizophrenic for long-time psychological test. In the event that a child with high curiosity is attracted by a picture, computer appears to be very patient to wait for the child's answering questions. In pen and paper-based test, however, it is easy to check the answers, but it is not the case in computer-aided test. Some researchers held that among the three methods of interview, computer-aided measurement and personal statement, testees prefer computer-aided measurement when facing sensitive topics.

Improvement of Test Efficiency

Immediately after the test is completed, computer will score and present a report. On the contrary, in the case of pen and paper-based test, the score needs to be manually calculated, the results need to be manually input, and a standard score is built according to a conventional model. This process consumes a lot of time, material resources and manpower. In computer-aided psychological measurement, the realization of rapid computation via software and hardware system largely saves material resources and manpower, and can give a score soon. It just takes a short time to develop a monitoring report. The group psychological measurement software developed by $\mathrm{Hu}$ Wendong can realize a large-scale psychological measurement process. Furthermore, computer is highly capable of data storage, greatly facilitating data storage for psychological measurement. Thus, researchers don't have to look up materials and data but just need call the materials they need via computer at any time. They also don't need to compute psychological data by themselves, which can be handled by computer.

\section{Limitations of Computer-aided Psychological Measurement}

Computer-aided psychological measurement has its limitations. For example, if the testee is not familiar with the operating procedure of computer and related specifications, his anxiety arising during test will influence the test result. 
It cannot completely substitute manual measurement.

Since computer is unemotional, it is unable to recognize the emotional fluctuation of the testee, nor answer questions of the testee. Thus, professionals are need to monitor the process of computer-aided test, who can handle any special circumstances in time. Besides, a test for subjective questions cannot be realized via computer. Take the example of Wechsler Adult Intelligence Scale, which is a highly subjective test. Hence, lots researches are still needed to realize such test via computer.

Testees' emphases vary.

In psychological test, testees are also an uncertain factor. They lay emphasis on different aspects. Due to interest difference of testees in psychological test, errors may occur, such as unconformity to reliability or validity. Through researches, Spinhoven found that it is less probable to obtain satisfactory results if the testees take a positive and relaxed attitude to computer-aided psychological measurement. Marcoulides' researches show that the anxiety about computer is mainly resulted by similar cultural type. Meanwhile, to avoid test inaccuracy due to the unacquaintance with computer of the testees, researchers designed some special answer inputting methods.

\section{Summary}

With the rapid development of computer and the Internet, psychologists take full advantage of computer that is featured by rapid processing speed, large information storage ability and high automation to maximize the speed of psychological measurement, and greatly improve the level of psychological measurement. Although computer is widely used in psychological measurement and clinical psychotherapy, there exist problems. Therefore, in computer-aided psychological measurement in future, moral issues should not be ignored while the development of computer is emphasized.

\section{References}

[1] Hu Wendong. Wang Tao. Li Xiaojing. Development of Computer Multi-media Group Psychological Measurement System. Journal of The Fourth Military Medical University.1999,20(3):

[2] Chen Guoming. Several Issues on the Application of Computer Technology in Psychological Test. Journal of Ningbo Institute of Education.1991.1

[3] Charles, Jackson. Know the Psychological Test Process. Translated by Yao Ping. Beijing: Peking University Press: 2000.35-46

[4] Qi Shuqing. Dai Haiqi. Ding Shuliang. Et al. Compilation and Application of Computerized Self-adapting Test. Jiangxi Educational Research:1999.2 